\title{
Centella asiatica (L.) extract attenuates inflammation and improve insulin sensitivity in a coculture of lipopolysaccharide (LPS)- induced 3T3-L1 adipocytes and RAW 264.7 macrophages
}

\author{
Siska Andrina Kusumastuti ${ }^{1}$, Dwi Aris Agung Nugrahaningsih ${ }^{2, *}$, \\ Mae Sri Hartati Wahyuningsih ${ }^{2}$ \\ ${ }^{1}$ Center of Pharmaceutical and Medicine Technology, Agency for the Assessment and Application of Technology (BPPT), Jakarta, \\ Indonesia; \\ ${ }^{2}$ Department of Pharmacology and Therapy, Medical Faculty, Universitas Gadjah Mada, Yogyakarta, Indonesia.
}

\begin{abstract}
Summary Insulin resistance in obese condition is related to chronic low-grade inflammation which leads to insulin signaling impairment. Centella asiatica (L.) is an herb that exhibits antiinflammatory and blood sugar-lowering activity (hypoglycemia). The study aims to investigate the molecular mechanism of $C$. asiatica extract in insulin sensitivity improvement in a coculture of lipopolysaccharide (LPS)-induced 3T3-L1 adipocytes and RAW 264.7 macrophages. A coculture of 3T3-L1 adipocytes and RAW 264.7 macrophages were incubated with LPS to induce insulin resistance in the adipocytes. An extract of $C$. asiatica was added to coculture cells and after $\mathbf{2 4}$ hours, insulin sensitivity and inflammatory response were determined, including glucose consumption, glucose transporter-4 (GLUT-4), insulin receptor substrate-1 (IRS-1), and interleukin-6 (IL-6) mRNA expression. $C$. asiatica extract at a concentration of $500 \mu \mathrm{g} / \mathrm{mL}$ increased glucose consumption and induced GLUT-4 and IRS-1 mRNA expression significantly in a coculture of LPS-induced 3T3-L1 adipocytes and RAW 264.7 macrophages. The pro-inflammatory cytokines IL-6 mRNA expression was decreased in the coculture cells after treatment with $C$. asiatica extract at a concentration of $500 \mu \mathrm{g} / \mathrm{mL}$. This result indicates that $C$. asiatica has an effect to stimulate glucose consumption in the coculture cells that might be mediated via GLUT-4/IRS-1 pathway as a result of IL-6 inhibition. These findings suggest that the $C$. asiatica extract inhibits inflammation and improves insulin sensitivity in a coculture of LPS-induced 3T3-L1 adipocytes and RAW 264.7 macrophages.
\end{abstract}

Keywords: Centella asiatica L., insulin sensitivity, coculture, 3T3-L1 adipocytes, RAW 264.7 macrophages

\section{Introduction}

Type 2 diabetes mellitus (T2DM) is a metabolic disorder caused by a defect in insulin action called insulin resistance (1). Insulin resistance in obesity is associated with chronic low-grade inflammation resulting from an increase of macrophages infiltration into adipose tissues (2). An interaction between excessive adipocytes and the higher amount of

\footnotetext{
*Address correspondence to:

Dr. Dwi Aris Agung Nugrahaningsih, Department of Pharmacology and Therapy, Medical Faculty, Universitas Gadjah Mada, Yogyakarta, Indonesia.

E-mail: dwi.aris.a@ugm.ac.id
}

macrophages promotes the macrophage activation into inflammatory macrophages (M2) followed by the secretion of pro-inflammatory cytokines such as interleukin-6 (IL-6), tumor necrosis factor- $\alpha$ (TNF- $\alpha$ ), and monocyte chemoattractant protein-1 (MCP-1) (3). Pro-inflammatory cytokines secreted from both adipocytes and activated macrophages may alter insulin sensitivity in adipocytes through the degradation and deactivation of insulin receptor substrate-1 (IRS-1), one of essential protein in the insulin receptor substrate-1/ phosphoinositide 3-kinases/Akt (IRS-1/PI3K/Akt) insulin signaling pathway $(3,4)$. The degradation of IRS-1 may trigger the downregulation of proteins downstream in the IRS-1/PI3K/Akt pathway, including glucose transporter-4 (GLUT-4) expression leading to 
the disruption of glucose uptake in adipocytes (5).

Anti-diabetic drugs efficacy in controlling blood glucose in diabetic patients has been established. However, they also have undesirable side effects such as weight gain, bone loss and increased cardiovascular risk (6). Therefore, safer and more effective alternative agents are needed and developing new drugs from herbal is a promising method.

Centella asiatica (L.) or Pegagan in Bahasa Indonesia is one of the most important herbal medicine used empirically in Indonesia. The herb has been proven to possess hypoglycemic activity in in-vivo models. Recent studies reported that $C$. asiatica extract decreased glucose plasma levels in high fat diet (HFD)induced and streptozotocin-induced diabetic mice in dose- dependent manner $(7,8)$. One of the active compounds in $C$. asiatica, asiatic acid has also been shown as an anti-inflammatory and a hypoglycemic agent $(10,11)$. Recent studies reported that asiatic acid decrease secretion of pro-inflammatory cytokines in the liver tissues of diabetic mice and inhibit LPSinduced inflammatory response in endometrial epithelial cells $(12,13)$. However, there is minimal information about molecular mechanism of $C$. asiatica extract in improving insulin sensitivity related to insulin resistance caused by inflammation. In the present study, we investigated the effect of $C$. asiatica extract on the insulin sensitivity of adipocytes using a coculture of LPS-induced RAW 264.7 macrophages and 3T3-L1 adipocytes. This study also evaluated involvement of the IRS-1/GLUT-4 pathway in relation to insulin sensitivity effect. The expression of the proinflammatory cytokine IL-6 was also investigated to determine $C$. asiatica effect on the inflammatory changes related to insulin sensitivity impairment.

\section{Materials and Methods}

\subsection{C. asiatica extract preparation}

A standardized C. asiatica extracts was produced by Javaplant PT, Tri Rahardja Tawangmangu Surakarta Indonesia, in September of 2016. Extraction was performed using water as a solvent and following good manufacturing practices. $C$. asiatica herbs were soaked in water at boiling point followed by stirring with a magnetic stirrer for $5 \mathrm{~h}$. Herbs were removed from the heat sources then filtered to obtain the concentrated extract. The freeze-drying method was used to obtain dried powder and then extract powder was dissolved in a Dimethyl Sulfoxide (DMSO) to prepare samples for testing.

\subsection{Cell lines and culture}

3T3-L1 preadipocyte cells and RAW 264.7 murine macrophage cells were obtained from the Food and
Science Technology Department of National Pingtung Science Technology (NPUST, Pingtung, Taiwan). Both cell lines were maintained in Dulbecco's modified Eagle's medium (DMEM, Gibco, New York, USA) containing $1 \%$ penicillin/streptomycin (PS, 100 units of penicillin/mL and $100 \mathrm{pg}$ streptomycin/mL, Gibco, New York, USA) and supplemented with 10\% New-born Calf Serum (NBCS, Gibco, New York, USA) to culture the 3T3-L1 cells and 10\% Fetal Bovine Serum (FBS, Gibco, New York, USA) to culture RAW 264.7 cells. The cells were incubated at $37^{\circ} \mathrm{C}$ in a humidified $5 \%$ $\mathrm{CO}_{2}$ atmosphere and the medium was replaced every 2 days.

\subsection{Viability assay}

RAW 264.7 macrophages were seeded at $2.4 \times 10^{4}$ cells/well and 3T3-L1 pre-adipocytes were seeded at $1.5 \times 10^{4}$ cells/well. Cells were cultured in a various concentrations $(62.5-1,000 \mu \mathrm{g} / \mathrm{mL})$ of the $C$. asiatica extract and then, the cells were incubated at $37^{\circ} \mathrm{C}$ in a humidified $5 \% \mathrm{CO}_{2}$ atmosphere for $24 \mathrm{~h}$. Viability assay of the 3T3-L1 pre-adipocyte cells and RAW 264.7 macrophage cells were performed using a previously described 3-[4,5- dimethylthiazol-2-yl]-2,5 diphenyl tetrazolium bromide (MTT) assay protocol (14). Safe concentrations of the extract were determined and used in further experiment.

\subsection{Coating the plates}

Gelatin 1.3\% (Sigma Aldrich, Saint Louis, USA) was dissolved in phosphate buffered saline (PBS). Transferred $2 \mathrm{~mL}$ of gelatin working solution into each well and then, plates were incubated at $37^{\circ} \mathrm{C}$ for $1 \mathrm{~h}$. The plates were dried for $30 \mathrm{~min}$ and sterilized by an Ultraviolet (UV) lamp for $15 \mathrm{~min}$.

\subsection{Differentiation and coculture of 3T3-L1 adipocyte cells and RAW 264.7 macrophage cells}

3T3-L1 pre-adipocytes were differentiated into mature adipocytes in gelatin-coated 6 well plate using a previously described protocol (15). RAW 264.7 macrophages were plated onto differentiated 3T3-L1 in serum-starved DMEM and incubated at $37^{\circ} \mathrm{C}$ in a humidified $5 \% \mathrm{CO}_{2}$ atmosphere for $3 \mathrm{~h}$. Lipopolysaccharide (Sigma Aldrich, Saint Louis, USA) at a concentration of $0.125 \mu \mathrm{g} / \mathrm{mL}$ was added to the cells in the FBS-supplemented DMEM medium and the cells were incubated at $37^{\circ} \mathrm{C}$ in a humidified $5 \%$ $\mathrm{CO}_{2}$ atmosphere for $48 \mathrm{~h}$. As a control, equal numbers of RAW 264.7 macrophages and differentiated 3T3-L1 cells were cultured separately in the same medium with a coculture. After $48 \mathrm{~h}, C$. asiatica extract dissolved in DMEM containing FBS $2 \%$ was added to a coculture cells at three different concentration $(125,250$ and 500 
$\mu \mathrm{g} / \mathrm{mL}$ ) followed by incubation at $37^{\circ} \mathrm{C}$ in a humidified $5 \% \mathrm{CO}_{2}$ atmosphere for an additional $24 \mathrm{~h}$. Cells were incubated in serum-starved DMEM containing $1 \mu \mathrm{g} / \mathrm{mL}$ insulin (Sigma Aldrich, Saint Louis, USA) for $30 \mathrm{~min}$. The medium and cells were collected and stored at $-20^{\circ} \mathrm{C}$ until use.

\subsection{Determination of glucose consumption}

Glucose concentration contained in the cell medium was measured using Glucose GOD FS glucose kit assay (Diasys Diagnostics, Holzheim, Germany) and was performed following the manufacturer's protocol with slight modifications. Reagents were added after $20 \mu \mathrm{L}$ of cells media was plated onto 96 well-plate. The plates were then incubated at $25^{\circ} \mathrm{C}$ for $20 \mathrm{~min}$. Absorbance at $540 \mathrm{~nm}$ was recorded using a microplate reader (Thermo Fisher Scientific Multiskan, Ratastie, Finland). All the samples were measured in triplicate.

\subsection{Quantitative of polymerase chain reaction}

A coculture of adipocytes and macrophages treated with the extract was harvested after $24 \mathrm{~h}$ of incubation. Control of RAW 264.7 macrophages and differentiated 3T3-L1 were harvested separately and then mixed in a single tube as a control without a coculture. Total RNA was isolated in each group according to manufacturer protocol (Geneaid biotech Ltd, New Taipei, Taiwan). Expression of GLUT-1, IRS-1 and IL-6 mRNA were measured by quantitative polymerase chain reaction (qPCR) using a Sensifast SYBR No-ROX One-Step Kit Master Mix Kit (Bioline, London, United Kingdom) and analyzed on an $\mathrm{Eco}^{\mathrm{TM}}$ Real-Time PCR instrument (Illumina Inc, San Diego, USA). The following primers were used for the qPCR: GLUT-4, 5'-TTGCACACGGCTTCCGAACG-3' (forward) and 5'-GATCTGCTGGAAACCCGACGG-3'; IRS-1 5'-CCATGAGCGATGAGTTTCGC-3' (reverse) and 5'- GCAGTGATGCTCTCAGTTCG; IL-6 5'-GAGTCACAGAAGGAGTGGCTAAG-3' (forward) and 5'-ACCACAGTGAGGAATGTCCAC-3' (reverse); and $\beta$-actin 5'-CTCTGGCTCCTAGCACCATGAAGA-3' (forward) and 5'-GTAAAACGCAGCTCAGTAAC AGTCCG-3' (reverse). The qPCR reaction cycling parameters for all genes were performed at $45^{\circ} \mathrm{C}$ for $10 \mathrm{~min}$ for reverse transcriptase activation, followed by a pre-incubation condition step at $95^{\circ} \mathrm{C}$ for $2 \mathrm{~min}$ and amplification for 40 cycles at $95^{\circ} \mathrm{C}$ for $5 \mathrm{sec}, 62^{\circ} \mathrm{C}$ for 15 sec and $72^{\circ} \mathrm{C}$ for $5 \mathrm{sec}$. Gene expression was calculated using the relative expression $\searrow \Upsilon \mathrm{Cq}$ method and $\beta$-actin was used as a normalizer. All samples were amplified in duplicate.

\subsection{Statistical analysis}

Data were expressed as the mean \pm SD. Statistical analysis were performed using the unpaired Student t-test and analysis of variance (ANOVA) followed by post-hoc tests. A value of $p<0.05$ was considered significant.

\section{Results}

3.1. Viability assay of the C. asiatica extract on 3T3-L1 preadipocytes and RAW 264.7 macrophages

To investigate $C$. asiatica extract effect on insulin sensitivity related to inflammatory changes in a coculture of differentiated 3T3-L1 and RAW 264.7 macrophages, we first evaluated its cytotoxicity at various concentration. Cells were treated with $C$. asiatica extract at concentrations up to $500 \mu \mathrm{g} / \mathrm{mL}$ showed viability more than $90 \%$ (Figure 1), suggesting that $C$. asiatica extract is safe and non-toxic up to a concentration of $500 \mu \mathrm{g} / \mathrm{mL}$ in both of 3T3-L1 preadipocytes and RAW 264.7 macrophages.

3.2 Coculture of RAW 264.7 macrophages in contact with 3T3-L1 adipocytes induced insulin resistance in adipocytes and altered macrophages morphology

Before we examine insulin sensitivity effect of $C$. asiatica extract on coculture cells, we evaluated adipocytes insulin responsiveness in a direct coculture of LPS-induced 3T3-L1 adipocytes and RAW 264.7 macrophages. Cellular contact in direct coculture under LPS treatment triggered morphological changes in the macrophages. RAW 264.7 macrophages appeared rounded without the LPS treatment and the macrophages became elongated with short extension appearance after LPS treatment. With 48 hours of the direct coculture, macrophages showed an elongated appearance with a long cellular extension between and

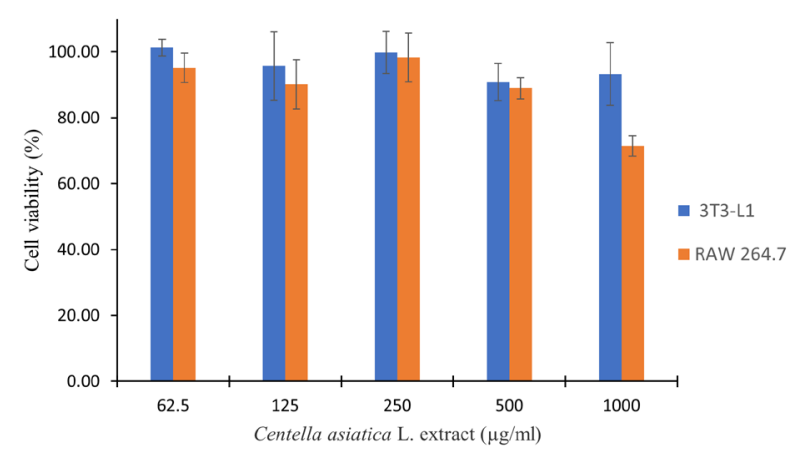

Figure 1. Effects of Centella asiatica (L.) extract on RAW 264.7 macrophages and 3T3-L1 pre-adipocytes viability. RAW 264.7 macrophages and 3T3-L1 preadipocytes were culturing in DMEM high glucose supplemented by New Calf Bovine Serum (NCBS) 10\% for culturing 3T3-L1 preadipocytes and Fetal Bovine Serum (FBS) 10\% for culturing RAW 264.7 macrophages in 96 well plate for $24 \mathrm{~h}$. The cells then were incubated at $37^{\circ} \mathrm{C}$ in a humidified $5 \% \mathrm{CO}_{2}$ with various concentration of Centella asiatica (L.) extract for $24 \mathrm{~h}$ and cell viability was determined using the MTT assay. Extract at a concentration up to $500 \mu \mathrm{g} / \mathrm{mL}$ showed cell viability more than $90 \%$ in both of the cells. Values are expressed as means \pm SD. 
A
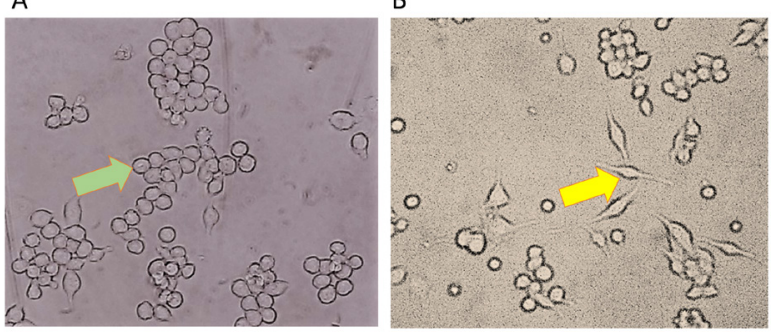

C

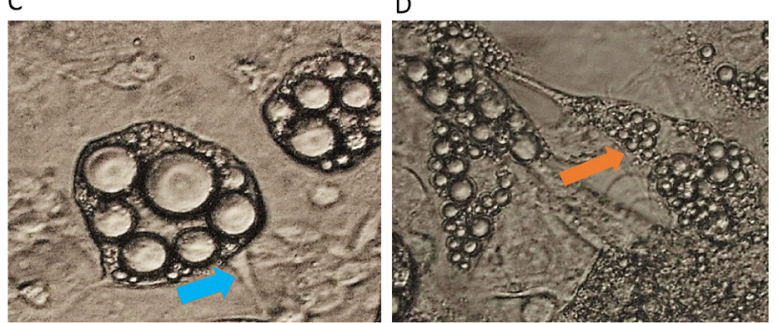

Figure 2. Morphological changes of RAW 264.7 macrophages without coculture and a direct coculture with adipocytes. Both of cells were culturing in DMEM high glucose supplemented by fetal bovine serum (FBS) $10 \%$ in 6 well plate and incubated at $37^{\circ} \mathrm{C}$ in a humidified $5 \% \mathrm{CO}_{2}$ for 48 h. Morphology of the cells were observed under microscope: (A) RAW 264.7 macrophages without LPS appeared small rounded (green arrow); (B) RAW 264.7 macrophages under LPS treatment showed elongated appearance with a short extension (yellow arrow); (C) Culture RAW 264.7 macrophages in contact with adipocytes under LPS treatment showed elongated appearance with a long cellular extension of RAW 264.7 macrophages between and along the surrounding adipocytes (blue arrow) and (D) Direct coculture between RAW 264.7 macrophages and differentiated 3T3-L1 adipocytes under LPS treatment developed lipid vacuoles accumulated in the macrophages cytoplasm (orange arrow).

along the surrounding adipocytes (Figure 2) resembling the morphology of adipose tissue macrophages (ATM) isolated from obese mice (16). However, direct contact between 3T3-L1 adipocytes and RAW 264.7 macrophages developed an accumulation of lipid vacuoles in the macrophages cytoplasm referred to as lipid-laden giant multinucleated. The results showed that a direct coculture of 3T3-L1 adipocytes and RAW 264.7 macrophages under LPS induction for $48 \mathrm{~h}$ stimulates changes in the macrophages behavior.

Insulin sensitivity was assessed by evaluating insulin-stimulated glucose consumption in a coculture of 3T3-L1 adipocytes and RAW 264.7 macrophages. Glucose concentration contained in a coculture medium indicates how much glucose was taken up by the coculture cells. Our study showed there were higher glucose concentration contained in the coculture of adipocytes and macrophages medium compared to the adipocytes medium only ( $p=0.046$, Figure $3 \mathrm{~A})$ indicating that an interaction between the adipocytes and macrophages under LPS treatment stimulated glucose uptake impairment in the adipocytes.

In this study, we determined insulin-stimulated GLUT-4 mRNA expression as a glucose transporter in adipocytes cells in contact with RAW 264.7 macrophages. We observed significantly lower GLUT-
A

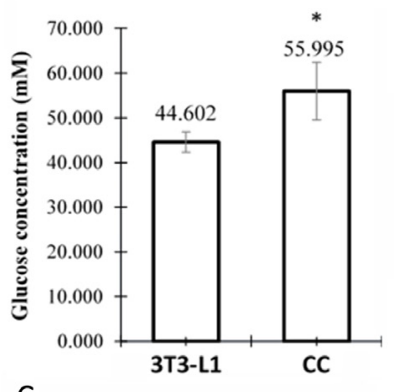

B
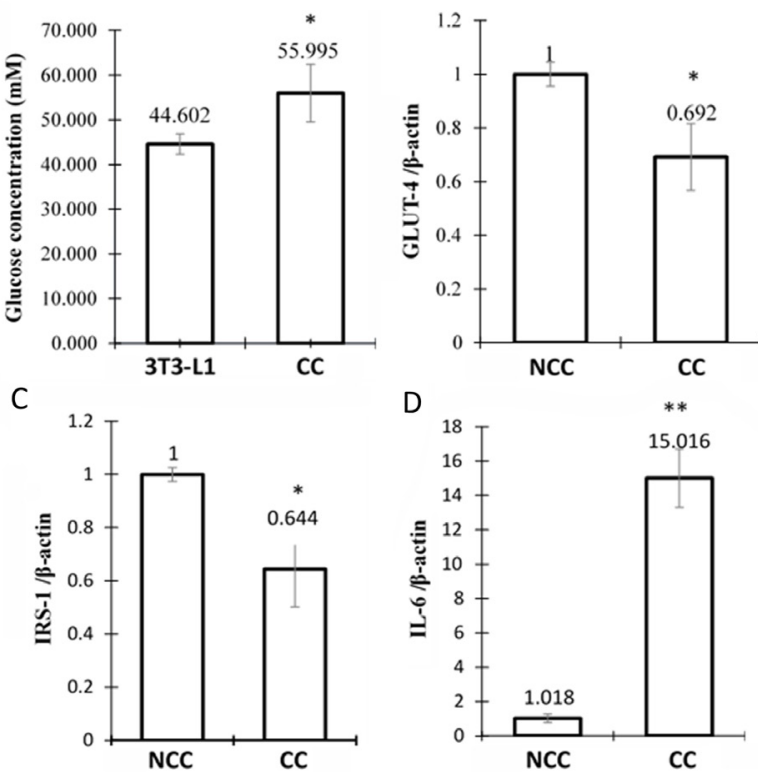

Figure 3. Insulin sensitivity and inflammatory response in control of coculture of LPS-induced adipocytes and macrophages (CC) and control of adipocytes and macrophages without co-culture (NCC). Parameters were measured after cells were incubated at $37^{\circ} \mathrm{C}$ in a humidified $5 \% \mathrm{CO}_{2}$ for $48 \mathrm{~h}$ and insulin induction for 30 minutes. (A) glucose concentration; (B) GLUT-4 mRNA; (C) IRS-1 mRNA; (D) IL-6 mRNA. Glucose concentration were measured using glucose assay kit and expression of target genes were quantified using qPCR. Values are mean \pm Standard Deviation. ${ }^{*} p<0.05$, vs. NCC; **p $<0.01, v s$. NCC.

4 mRNA expression in a coculture of LPS-induced 3T3-L1 adipocytes and RAW 264.7 macrophages compared to the sum of the GLUT-4 mRNA expression by adipocytes and macrophages without a co-culture $(p$ $=0.016$, Figure $3 \mathrm{~A}$ ). This result is consistent with the glucose consumption data previously.

Glucose transport involves insulin signaling pathways after insulin stimulation. The IRS1/PI3K/Akt pathway is essential for insulin-regulated glucose transport by activating and translocating GLUT-4 from intracellular vesicles to the plasma membrane. This study determined the mRNA expression of IRS-1. The result showed that IRS-1 mRNA expression in coculture of LPS-induced adipocytes and macrophages was significantly lower $(p=0.045)$ compared to the sum of the IRS-1 mRNA expression by adipocytes and macrophages without a coculture (Figure 3C).

To evaluate inflammatory changes caused by the interaction between adipocytes and macrophages under LPS treatment, we assessed pro-inflammatory cytokine IL-6 mRNA expression in a coculture of 3T3L1 adipocytes and RAW 264.7 macrophages. The coculture cells showed significantly higher of IL-6 mRNA expression compared to the sum of the IL-6 mRNA expression by adipocytes and macrophages without a co-culture $(p<0.01$, Figure 3D). This result suggested that direct contact between adipocytes and macrophages under LPS treatment induced an 
A

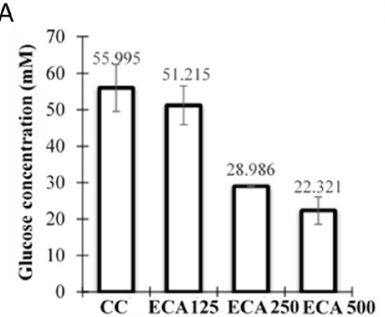

C

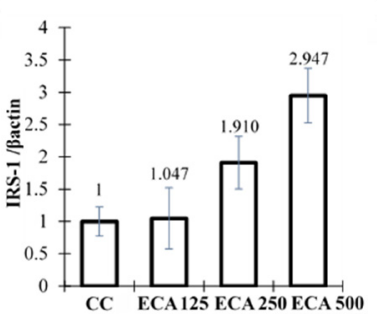

B

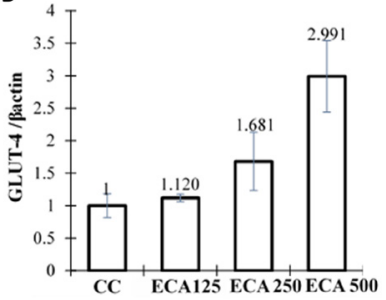

D

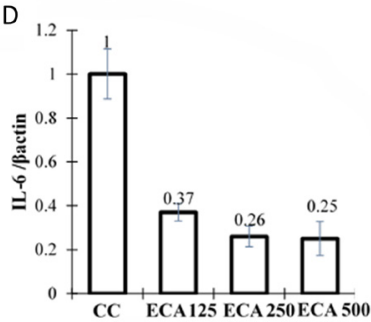

Figure 4. Insulin sensitivity and inflammatory response in control of coculture (CC) and coculture after treatment with Centella asiatica (L.) extract (ECA). Parameters were measured after both of cells incubated with a various concentration of Centella asiatica L. extract $(125,250$, and 500 $\mu \mathrm{g} / \mathrm{mL}$ ) at $37^{\circ} \mathrm{C}$ in a humidified $5 \% \mathrm{CO}_{2}$ for $24 \mathrm{~h}$, followed by insulin induction for $30 \mathrm{~min}$. (A) glucose concentration; (B) GLUT-4 mRNA; (C) IRS-1 mRNA; (D) IL-6 mRNA. Glucose concentration were measured using glucose assay kit and expression of target genes were quantified using qPCR. Values are mean \pm Standard Deviation. $* p<0.05$, vs. CC; $* * * p<0.001$, vs. CC.

inflammatory response, which in turn, influence the adipocytes insulin sensitivity that might have mediated via the IRS-1/GLUT-4 pathway.

\subsection{Glucose consumption in a coculture of LPS-induced 3T3-L1 adipocytes and RAW 264.7 macrophages after $24 \mathrm{~h}$ treatment of $C$. asiatica extract}

To evaluate $C$. asiatica extract effect to improve insulin sensitivity, we determined glucose concentration contained in a coculture of LPS-induced adipocytes and macrophages medium after treated by $C$. asiatica extract at a various concentration $(125,250$, and 500 $\mu \mathrm{g} / \mathrm{mL}$ ) for $24 \mathrm{~h}$. Glucose concentration contained in a coculture LPS-induced of adipocytes and macrophages medium after treated with $C$. asiatica extract at concentration 250 and $500 \mu \mathrm{g} / \mathrm{mL}$ showed significantly lower concentrations compared to a differentiated adipocytes in a dose-dependent manner $(p<0.01$ and $p$ $<0.01$, respectively, Figure $4 \mathrm{~A}$ ). This result indicated that $C$. asiatica extract improved glucose consumption in a coculture cells.

3.4. GLUT-4 and IRS-1 mRNA expression in a coculture of LPS-induced 3T3-L1 adipocytes and RAW 264.7 macrophages after a $24 \mathrm{~h}$ treatment with $C$. asiatica extract

A co-culture of adipocytes and macrophages treated with $C$. asiatica extract at a concentration of $500 \mu \mathrm{g} /$

mL showed significantly higher GLUT-4 and IRS-1 mRNA expression compared to a control of coculture of adipocytes and macrophages $(p<0.01$ and $p<$ 0.01 , respectively) as shown in Figures $4 \mathrm{~B}$ and $4 \mathrm{C}$. This result suggested that improvement of glucose consumption in a coculture cells after treated with $C$. asiatica extract at a concentration of $500 \mu \mathrm{g} / \mathrm{mL}$ might be related to the upregulation of GLUT-4 and IRS-1 mRNA expression in the adipocytes.

\subsection{Pro-inflammatory cytokine IL-6 $m R N A$ expression in a coculture of LPS-induced 3T3-L1 adipocytes and $R A W 264.7$ macrophages after $24 \mathrm{~h}$ treatment with $C$. asiatica extract}

A direct interaction between adipocytes and macrophages aggravates inflammatory changes in adipose tissue marked by the increased production of pro-inflammatory cytokines such as IL-6, TNF- $\alpha$ and IL-1 $\beta$. This study determined pro-inflammatory cytokine IL-6 mRNA expression in a coculture of LPSinduced adipocytes and macrophages after treatment with $C$. asiatica extract. We observed that extract at a concentration of $500 \mu \mathrm{g} / \mathrm{mL}$ attenuates inflammatory response in cells marked by significantly lower IL-6 mRNA expression in a coculture of adipocytes and macrophages compared to control of coculture of adipocytes and macrophages as shown in Figure 4D ( $p$ $=0.026)$.

\section{Discussion}

C. asiatica is a promising herb with anti-inflammatory and hypoglycemic activity. This study aimed to investigate the effect of $C$. asiatica extract on insulin sensitivity improvement related to the inflammatory response and its insulin signaling mechanism in a coculture of LPS-induced 3T3-L1 adipocytes and RAW 264.7 macrophages.

Direct contact between adipocytes and macrophages stimulates cytokines pro-inflammatory production such as IL-6, TNF- $\alpha$, MCP-1, and IL- $1 \beta$ which may, in turn alter insulin sensitivity in adipocytes $(16,17)$. In this study, a direct coculture of 3T3-L1 adipocytes and RAW 264.7 macrophages treated with TLR-4 lipopolysaccharide (LPS) showed that direct contact between adipocytes and macrophages under LPS induction stimulated insulin resistance in the adipocytes. A coculture of adipocytes and macrophages showed glucose consumption impairment followed by degradation of GLUT-4 and IRS-1 mRNA. The coculture of adipocytes and macrophages under LPS induction also elevated IL-6 mRNA expression compared to the sum of IL- 6 expression in adipocytes and macrophages without a co-culture. This result suggested that upregulated of $I L-6$ gene expression caused by direct contact and LPS induction in a 
coculture cells might lead to IRS-1 degradation resulting in GLUT-4 downregulation. GLUT-4 as a glucose transport is a key protein in the regulation of glucose in adipose tissue thus decreasing GLUT4 mRNA expression should affect insulin sensitivity in adipocytes. It means that higher expression of proinflammatory cytokines in adipocyte tissue plays a pivotal role in the disruption of insulin sensitivity.

Insulin signaling impairment caused by proinflammatory cytokines might be influenced by upregulation of suppressor of cytokine signaling (SOCS)1/3 protein. A study conducted by Rui found that the activation and upregulation of the SOCS1/3 protein caused by pro-inflammatory cytokines altered insulin sensitivity in inflammation-induced adipocytes that have been shown to reduce IRS1/2 proteins by targeting them through ubiquitin-mediated proteasomal degradation (18).

C. asiatica extract at a concentration of $500 \mu \mathrm{g} / \mathrm{mL}$ was shown to improve insulin sensitivity in a coculture of LPS-induced 3T3-L1 adipocytes and RAW 264.7 macrophages. Increasing glucose consumption in a coculture of LPS-induced adipocytes and macrophages after treatment with the extract at a concentration of $500 \mu \mathrm{g} / \mathrm{mL}$ was followed by upregulation of GLUT4 and $I R S-1$ gene expression in adipocytes. This result indicates that upregulation of GLUT-4 and IRS-1 gene expression in a coculture cells after treated with extract at a concentration of $500 \mu \mathrm{g} / \mathrm{mL}$ might be contributed to insulin sensitivity improvement in adipocytes.

C. asiatica extract at a concentration of $500 \mu \mathrm{g} / \mathrm{mL}$ attenuated inflammatory response in a coculture of LPSinduced 3T3-L1 adipocytes and RAW 264.7 macrophages by inhibiting the pro-inflammatory cytokine IL-6. This finding is consistent with the gene expression of GLUT4/IRS-1 and the glucose consumption results, indicating that the effect of $C$. asiatica extract to improve insulin sensitivity might be mediated through the IRS-1/GLUT4 pathway as a result of inhibiting the pro-inflammatory cytokine IL-6.

Recent studies reported that leaf aqueous extract of $C$. asiatica at a concentration of $50 \mathrm{mg} / \mathrm{kg}$ of body weight has a glucose-lowering effect in alloxan-induced diabetic rats compared to diabetic and anti-diabetic drug groups (19). Other studies have also shown that an ethanol extract of $C$. asiatica has an anti-diabetic effect at a concentration of $200 \mathrm{mg} / \mathrm{kg}$ of body weight based on blood-glucose serum level compared to the control group (20). However, asiatic acid, as an active compound in C. asiatica, has anti-inflammatory activity by downregulating the pro-inflammatory cytokines IL6 , IL- $1 \beta$, and TNF- $\alpha$ in human corneal epithelial cells and the liver tissue of diabetic-induced mice $(12,13)$. In addition, asiatic acid exerts anti-hyperglycemia effect in high fat diet (HFD)-induced diabetes mice through the PI3K/Akt/GSK $\beta$ signaling pathway (21). Another previous study showed that asiatic acid at a dose of 20 $\mathrm{mg} / \mathrm{kg}$ body decreased blood-glucose level in diabeticinduced rat using streptozotocin (STZ) followed by improved glucose uptake into skeletal muscle tissues via the IRS-1/PI3K-Akt signaling pathway (22).

IRS-1 is a key intracellular molecule that mediates insulin signaling through the IRS-1/PI3K/Akt pathway. Defects in or degradation of IRS-1 in adipocytes may be causes of insulin resistance (23). IRS-1 gene disruption in obese mice impaired glucose transports, suggesting that insulin sensitivity in adipocytes is dependent on the expression of the IRS-1 protein (2426). Upregulated $I R S-1$ gene expression after treatment with $C$. asiatica at a concentration of $500 \mu \mathrm{g} / \mathrm{mL}$ might be related to its inflammation inhibition effect inducing macrophages to polarize into "macrophage alternatively activated" M2. A study conducted by Hawas found that treatment with $C$. asiatica at doses of 500 and 100 $\mathrm{mg} / \mathrm{kg}$ of body weight prevented M1/M2 ratio from increasing in diabetic rats (27). This effect might trigger the deactivation of c-Jun-NH terminal kinase (JNK) and decrease SOC1/3 expression which in turn affects IRS-1 upregulation. Increasing GLUT-4 and IRS-1 gene expression in adipocytes after treatment with $C$. asiatica might contribute to the adipocytes ability to take up glucose upon insulin stimulation.

In conclusion, $C$. asiatica extract alleviated the inflammatory response by inhibiting the proinflammatory cytokine IL- 6 and improved insulin sensitivity in adipocytes marked by stimulated glucose consumption in a coculture cells that might be mediated via IRS-1/GLUT-4 insulin signaling pathway. However, further studies are required to evaluate more fully understanding about molecular actions of $C$. asiatica extract in insulin sensitivity improvement in adipocytes related to inflammatory changes.

\section{Acknowledgments}

We thank Professor Huang from the Department of Biological Science at NPUST Taiwan for providing us with the 3T3-L1 cells and RAW 264.7 cells as well as the Molecular Biology and Cell Culture Laboratory at LAPTIAB BPPT for supporting this experiment by providing access to its facilities and reagents.

\section{References}

1. Scheen A. Pathophysiology of type 2 diabetes. Acta Clin Belg. 2014; 110:335-341.

2. Sun K, Kusminski C, Scherer P. Adipose tissue remodeling and obesity. J Clin Invest. 2011; 121:20942101.

3. Pahlavani M, Ramalho T, Koboziev I, Lemieux M, Jayarathne S, Ramalingam L, Filgueiras L, MoustaidMoussa N. Adipose tissue inflammation in insulin resistance: Review of mechanisms mediating antiinflammatory effects of omega-3 polyunsaturated fatty acids. J Investig Med. 2017; 65:1021-1027. 
4. Rotter V, Nagaev I, Smith U. Interleukin-6 (IL-6) induces insulin resistance in 3T3-L1 adipocytes and is, like IL-8 and tumor necrosis factor- $\alpha$, overexpressed in human fat cells from insulin-resistant subjects . J Biol Chem. 2003; 278:45777-45784.

5. Pederson T, Kramer D, Rondinone C. Serine/threonine phosphorylation of IRS-1 triggers its degradation. Diabetes. 2001; 50:24-31.

6. Piera-Mardemootoo C, Lambert P, Faillie JL. Efficacy of metformin on glycemic control and weight in drug-naive type 2 diabetes mellitus patients: A systematic review and meta-analysis of placebo-controlled randomized trials. Therapie. 2018. pii: S0040-5957(18)30019-2.

7. Mutayabarwa C, Sayi J, Jande M. Hypoglycaemic Activity of Centella Asiatica (L) Urb. East Cent. Afr J Pharm Sci. 2003; 6:30-35.

8. Gayathri V, Lekshmi P, Padmanabhan R. Anti-diabetes activity of ethanol extract of Centella asiatica (L.) Urban (whole plant) in streptozotocin-induced diabetic rats, isolation of an active fraction and toxicity evaluation of the extract. Int J Med Arom Plants. 2011; 1:278-286.

9. Sasikala S, Lakshminarasaiah S, Naidu M. Antidiabetic activity of Centella asiatica on streptozotocin induced diabetic male albino rats. World J Pharm Sci. 2015; 3:1701-1705.

10. Hashim P, Sidek H, Helan M, Sabery A, Palanisamy U, Ilham M. Triterpene composition and bioactivities of Centella asiatica. Molecules. 2011; 16:1310-1322.

11. Chen H, Hua X, Ze B, Wang B, Wei L. The anti-inflammatory effects of asiatic acid in lipopolysaccharide-stimulated human corneal epithelial cells. Int J Opthalmol. 2017; 10:179-185.

12. Oyenihi A, Chegou N, Oguntibeju O. Centella asiatica enhances hepatic antioxidant status and regulates hepatic inflammatory cytokines in type 2 diabetic rats. Pharm Biol. 2017; 55:1671-1678.

13. Cao SY, Wang W, Nan FF, Liu YN, Wei SY, Li FF, Chen L. Asiatic acid inhibits LPS-induced inflammatory response in endometrial epithelial cells. Microb Pathog. 2018; 116:195-199.

14. Twentyman P, Luscombe M. A study of some variables in a tetrazolium dye ( MTT ) based assay for cell growth and chemosensitivity. Br J Cancer. 1987; 56:279-285.

15. Zebisch K, Voigt V, Wabitsch M, Brandsch M. Protocol for effective differentiation of 3T3-L1 cells to adipocytes. Anal Biochem. 2012; 425:88-90.

16. Lumeng C, Deyoung S, Saltiel A. Macrophages block insulin action in adipocytes by altering expression of signaling and glucose transport protein. Am J Physiol Endocrinol Metab. 2007; 292:1-23.

17. Nakarai H, Yamashita A, Nagayasu S, Iwashita M,
Kumamoto S, Ohyama H, Hata M, Soga Y, Kushiyama A, Asano T, Abiko Y, Nishimura F. Adipocytemacrophage interaction may mediate LPS-induced low-grade inflammation: Potential link with metabolic complications. Innate Immun. 2012; 18:164-170.

18. Rui L, Yuan M, Frantz D, Shoelson S, White MF. SOCS1 and SOCS-3 block insulin signaling by ubiquitinmediated degradation of IRS1 and IRS2. J Biol Chem. 2002; 277:42394-42398

19. Emran T, Dutta M, Uddin M, Nath, A, Uddin, Md. Antidiabetic potential of the leaf extract of Centella asiatica in alloxan-induced diabetic rats. Jahangirnagar Univ J Biol Sci. 2015; 4:51-59.

20. Gayathri V, Lekshmi P, Padmanabhan R. Anti-diabetes activity of ethanol extract of Centella asiatica ( L .) Urban (whole plant) in streptozotocin-induced diabetic rats, isolation of an active fraction and toxicity evaluation of the extract. Int J Med Arom Plants. 2011; 1:278-286.

21. Sun W, Xu G, Guo X, Luo G, Wu L, Hou Y, Guo X, Zhou J, Xu T, Qin L, Fan Y, Han L, Matsabisa M, Ma X, Liu T. Protective effects of asiatic acid in a spontaneous type 2 diabetic mouse model. Mol Med Rep. 2017; 16:1333-1339.

22. Ramachandran V, Saravanan R. Glucose uptake through translocation and activation of GLUT4 in PI3K/Akt signaling pathway by asiatic acid in diabetic rats. Hum Exp Toxicol. 2015; 34:884-893.

23. Hoehn K, Hohnen-behrens C, Cederberg A, Wu L, Turner N, Yuasa T, Ebina Y, James D. IRS1-independent defects define major nodes of insulin resistance. Cell Metab. 2010; 7:421-433.

24. Araki E, Lipes M, Patti M, Bruning J, Haag BL III, Johnson RS, Kahn C. Alternative pathway of insulin signaling in mice with targeted disruption of the IRS-1 gene. Nature. 1994; 372:186-190.

25. Tamemoto H, Kadowaki $\mathrm{T}$, Tobe $\mathrm{K}$, et al. Insulin resistance and growth retardation in mice lacking insulin receptor substrate-1. Nature. 1994; 372:182-186.

26. Patti M, Sun X, Bruning J, Araki E, Lipes M, White M, Kahn C. 4PS/IRS-2 is the alternative substrate of the insulin receptor in IRS-1 deficient mice. J Biol Chem. $1995 ; 270: 24670-24673$.

27. Hawas A., Nugrahaningsih A, Solikhah E, Syarifuddin A, Wijayaningsih R, Ngatidjan, Anti-inflammatory effect of Centella asiatica L. extract on prevented aortic intimamedia thickening in diabetic rats. J Pharm Sci. 2017; $41: 1-7$.

(Received July 8, 2019; Revised August 24, 2019; Accepted October 22, 2019) 